\title{
NEW CONTRIBUTIONS ON THE DOME OF THE PANTHEON IN ROME: COMPARISON BETWEEN THE IDEAL MODEL AND THE SURVEY MODEL
}

\author{
L. Aliberti ${ }^{\text {a, }}$, M. Canciani ${ }^{\text {b }}$, M.A. Alonso Rodriguéz ${ }^{\mathrm{c}}$ \\ ${ }^{a}$ Departamento de Ideación Gráfica Arquitectónica, Universidad Politécnica de Madrid - licinia.aliberti@gmail.com \\ ${ }^{b}$ Dipartimento di Architettura, Università degli Studi Roma Tre - marco.canciani@uniroma3.it \\ ${ }^{\mathrm{c}}$ Departamento de Ideación Gráfica Arquitectónica, Universidad Politécnica de Madrid - miguel.alonso@upm.es
}

Commission V, WG V/4

KEY WORDS: Pantheon, integrated survey, ideal model, survey model, surface

\begin{abstract}
:
This work proposes an integrated survey and a study of the intrados of the dome of the Pantheon in Rome. An actualized architectural survey of the interior of the dome can generate useful material for future studies.

The survey has been realized by using in a first stage the digital photogrammetry and in a second stage the three-dimensional laser scan technology. The compared analysis between different methods applied in the same object is useful towards a closer approximation to real dimension.

Among several aspects that arise in dealing with the Pantheon this work focuses mainly on the study of the geometry of the inner surface of the dome. The specific goal of the research is to verify the spherical form of the surface and the coffers' distribution. In this sense it takes an important place the extracting data system. In order to realize the analysis it was applied a critical treatment of selected information contained in the point cloud. The use of plan and section drawings connects to the study of three dimensional models.

The research is based on the construction of an ideal geometrical model that derives from the theoretical model described in the historical documents. The survey points model, which keeps the irregularities of the actual form, determines the creation of an average sphere, that is a regular model defined by clarifying geometrical laws.

The direct comparison between the survey model and the ideal model contributes to the building understanding. It detects irregularities or deformities where they exist, and provides objective and quantifiable data.
\end{abstract}

\section{INTRODUCTION}

This work proposes an integrated survey and a study of the intrados of the dome of the Pantheon in Rome, based on a PHD research (Aliberti 2014). The intense study of such surprising architecture lasts over the centuries and the monument still offers unsolved questions. An actualized architectural restitution of the interior of the dome can generate useful material for future studies, considering that the last surveys of the building were published by Belardi (2006), Valenti (2009) and Graßhoff (2009).

Among several aspects that arise in dealing with the Pantheon this research focuses mainly on the study of the geometry of the inner surface of the dome. The constructive solutions applied by roman constructors contribute to realize this singular and complex architecture that is still completely conserved. The specific goal of the research is to verify the spherical shape of the surface and the coffers' distribution.

The different analysis developed show the importance of integrating the building metric restitution with the study of its history, geometry and construction. The survey is considered as a comprehensive system of architectural understanding. Bibliographic documentation enriches the research indicating where and when to look in a sharper way.

Nevertheless the metric restitution of the dome may be considered as the first necessary step to develop the research. The integrated survey realized using a $3 \mathrm{D}$ laser scan and the digital photogrammetry offers complete and detailed information about the inner surface of the dome.
In order to carry out the geometrical analysis of the dome inside shape, a critical study is applied to data derived from surveys. The research is based on the construction of a geometrical model that approximates the survey points and that is generated with a rigorous average calculation. Survey points, which keeps the irregularities of the constructed object, determines the creation of a regular model defined by clarifying geometrical laws. The direct comparison between the individual positions of the survey points and the geometrical model contributes to the building understanding. By this method we can detect irregularities or deformities where they exist, and provide objective and quantifiable data.

The study of the survey results and their comparison with the ideal model was possible due to a specific data treatment. In order to recognize the specific shape of the object it is necessary to select data from the large information of the point cloud. This process consists in several steps starting from the creation of a continuous surface until the extraction of planar sections. The simplification of the point cloud into a triangulated surface may generate a loss of information. Then in some specific studies, the analysis of part of the dome was realized generating section curves directly from the point cloud. This method can be a further step in the study of the 3D survey in the architectural field (Canciani et al. 2013).

The bi-dimensional drawing allows to study the implicit complexity of architecture by isolating some of its parts and operating in a reduced environment. In this work, the plan and section drawings have been considered as a necessary tool to develop the geometrical analysis. 
At the same time it was realized a comparison study directly working into the three-dimensional work space of vectorial drawing software. These operations have been especially useful in order to realize the study of the continuous surface of the intrados of the dome. The observation of the 3D model permitted the use of calculations of the relative positions of the survey points so that it was possible to determine their distances from the ideal sphere. Furthermore we could detect and indicate the position and the extension of the deformation areas of the intrados of the dome.

The integrated studies realized using plan and section drawings and the three-dimensional models improved the research and generated useful information about the present condition of the Pantheon dome.

\section{SURVEY MODEL AND IDEAL MODEL}

\subsection{Survey model}

The complete survey of the intrados of the dome was obtained through three-dimensional laser scanner and a digital photogrammetric restitution. Integrated softwares enabled the comparison of results and verified the accuracy of surveys. By analysing the outcome we can state that the results in both methods are coherent and complementary in a certain way. A contrast between different versions of restitution of the same object is useful for a better approximation to the real shape.

The photogrammetric survey was realized using different series of photos and comparing the results (Fig. 1). The inner surface of the dome is completely visible and it can be photographed from different points, except for the hidden area covered by the cornice that separates the drum from the dome. The existence of coffers takes a great importance in the visual perception of the dome and contributes to reconstruct the spherical shape of the surface; meanwhile they are useful in this work of metric restitution as evident reference elements.

The photographic camera used to catch the images was a fullframe digital reflex, with fixed-focus lens that were changed according to need. It is relevant the circumstance that it was used a high definition camera to make the photographs. The software used to realize the photogrammetric restitution is the Photo Modeler Scanner and the point model obtained was analyzed with common vectorial drawing softwares like AutocCAD and Rhinoceros.

Different restitution models were generated applying a selection of photo series with diverse topics. Successively it was selected the model with a better approximation and photo orientation. This model includes all internal and external points of the coffers, several points of the cornice that separates the tambour from the dome and some other points that describe the oculus. The points selection was possible due to the coffers' shape and to the marble joints of the cornice (Fig. 2). However some difficulties where found in the restitution of the continuous surface of the intrados of the dome. Therefore it was introduced another survey method.

The 3D scanner survey was realized in the summer of 2012 using a Leica HD6200 scanner (Fig. 3). The data were collected in a short interval of time because of the use restrictions of the monument.

The scanner was positioned in several places. The data works include five scanning stations. One of them was realized in high definition from the centre of the space. Other four scanning sessions were developed from different places using a reduced definition. The range registration and alignment was executed using the point cloud data management software Leica Cyclone.

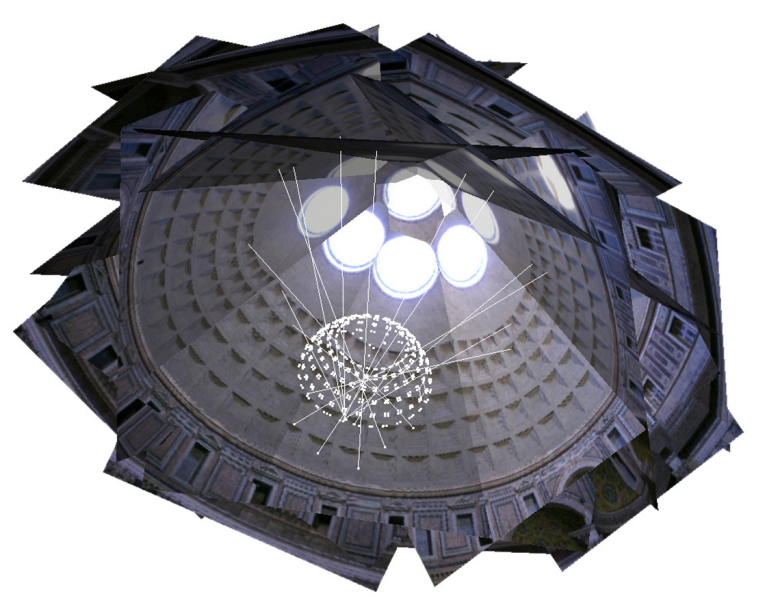

Figure 1. View of the photogrammetric model and the oriented photos used for the restitution.

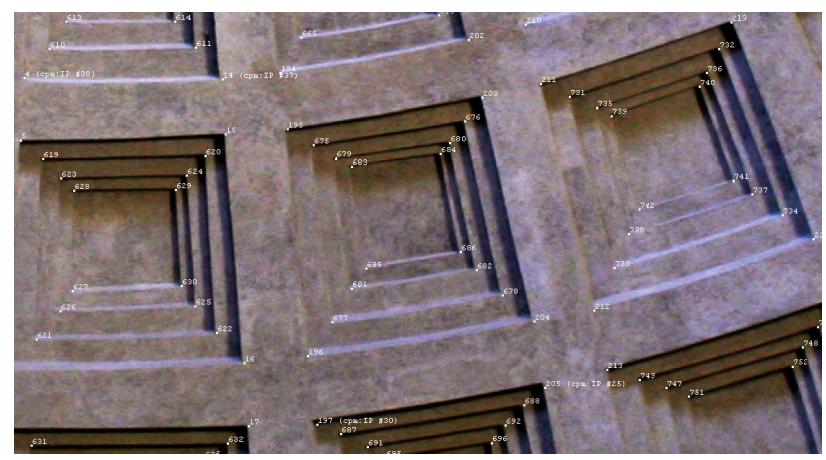

Figure 2. View of vertices points obtained by photogrammetry.

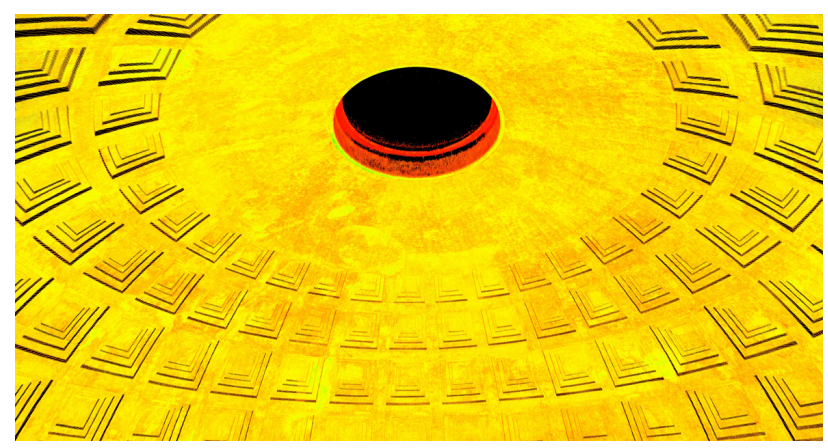

Figure 3. View of the point cloud.

The central scan of the inner space describes all the intrados of the dome including the coffers' area without any shadow. All the interior levels and the edges are visible from the center of the space, where these elements can be appreciated with a better perception (Licht 1968; Lucchini 1996; Waddell 2008). This fact shows directly the geometrical qualities of Pantheon's coffers. Their design follows complex projective laws. 
In order to carry out the research, a critical study was applied to the outcome of the survey. The necessary information for realizing the geometrical analysis was extracted from the point cloud. The general model obtained from the different scenes alignments was exported to the software 3DReshaper, that offers useful tools to realize a sharp study of the point cloud. Using this software, a continuous mesh surface was generated from the point cloud as a best fit result.

The extraction of a surface from the point cloud is an essential condition to the research development. These procedures, in fact, are aimed to realize the geometrical analysis of the intrados of the dome, that requires a work with continuous elements like curves and surfaces (Docci \& Maestri 2010). In the study of the coffers distribution the photogrammetric model has been very useful as a complementary tool. The manual selection of points in the restitution process generates a clear definition of the coffers vertices. On the other hand the triangulation of the mesh surface brings some problems in the identification of the vertices. The use of points from photogrammetric method with those selected from the scanner point cloud allows the study of the coffers' general distribution.

\subsection{Ideal model}

Historical surveys and documents used to represent the intrados of the Pantheon's dome like a perfect hemisphere, with the center situated in the cornice line that divides the drum from the vault. A specific study in the actual spherically-shaped condition of this surface would reconnect to the theoretical model of Pantheon.

To make possible the reconstruction the ideal geometry of the intrados of the dome, we have studied the sphere to which the points of the cloud approximate. The ideal sphere gets close to the survey points and is generated with a rigorous average calculation.

In order to calculate the radius and position of the average sphere, the works have been developed in two ways: either by using a selection of the points, or by using the entire points cloud.

The resulting mesh surface has been cut in 20 horizontal sections and 28 vertical sections. 14 of the vertical sections have been produced on the ribs and the other 14 on the axis of the coffers. The intersection points of the horizontal and vertical sections were used for the informatics calculations of the average sphere. These calculations have been realised with the software Rhinoceros under two different conditions. One is considering all the points resulting in a sphere of radius 21.95 meters. The other considers all the points except those of the upper area and results in a radius value of 22.03 meters. The upper part of the dome's model contains a bigger number of horizontal curves. The points in this part show a depression that decreases the value of the resultant radius.

The subsequent studies have been realized by comparing the results with the radius $22.03 \mathrm{~m}$ sphere with no deformations.

The diameter of this calculus matches the result of 44.06 meter obtained by the points cloud management program 3DReshaper (best fit). This operation manages a big number of points uniformly distributed.

In this way we obtain a double verification of the accuracy of the average value, by considering the entire cloud in a specific management program, and by considering just some well selected points in the model and proceeding to their analysis with vectorial standard software. The average calculated model could be taken as the ideal sphere.

Most of the publications on the Pantheon describe a value for the diameter of the dome between 43.30 and 43.80 meters, although specific recent studies like Pelletti's (1989), show a value of 22.03 that matches the achieved results. According to a recent survey carried out by the University "La Sapienza" in Rome (Valenti 2009) the average radius is close to 21.90 meters in the coffers' part while 21,40 in the oculus part. The laser scanner survey carried out in 2009 by the Bern University (Graßhoff 2009), registers a radius of $21.99 \mathrm{~m}$, a gap of $4 \mathrm{~cm}$ to the present study.

According to the classic descriptions of the Pantheon, the centre of the dome belongs to the plan of the cornice between the drum and the dome. This fact is collected in the historical surveys from the publications of the Renaissance to the first rigorous study by Desgodetz (1682) and the later representations. Only in the recent surveys another position is set for the centre of the interior sphere.

This work like Licht (1968), Pelletti (1989) or Graßhoff (2009), determines the existence of an offset between the cornice plan and the centre of the inscribed sphere. In order to reconstruct the cornice line it was applied an average calculation to the survey points. The circumference line obtained belongs to a plan forming 0.44 degrees with the horizontal plan. It was fixed a reference height in the horizontal plan passing through the centre of the average circumference of the cornice. The position of the centre of the ideal sphere is calculated at $-0,37 \mathrm{~m}$ behind this reference system based on the reconstruction of the cornice line that divides the drum from the dome (Fig. 4). This feature suggests a first difference between the ideal geometric model and the theoretical model described in the historical documents.

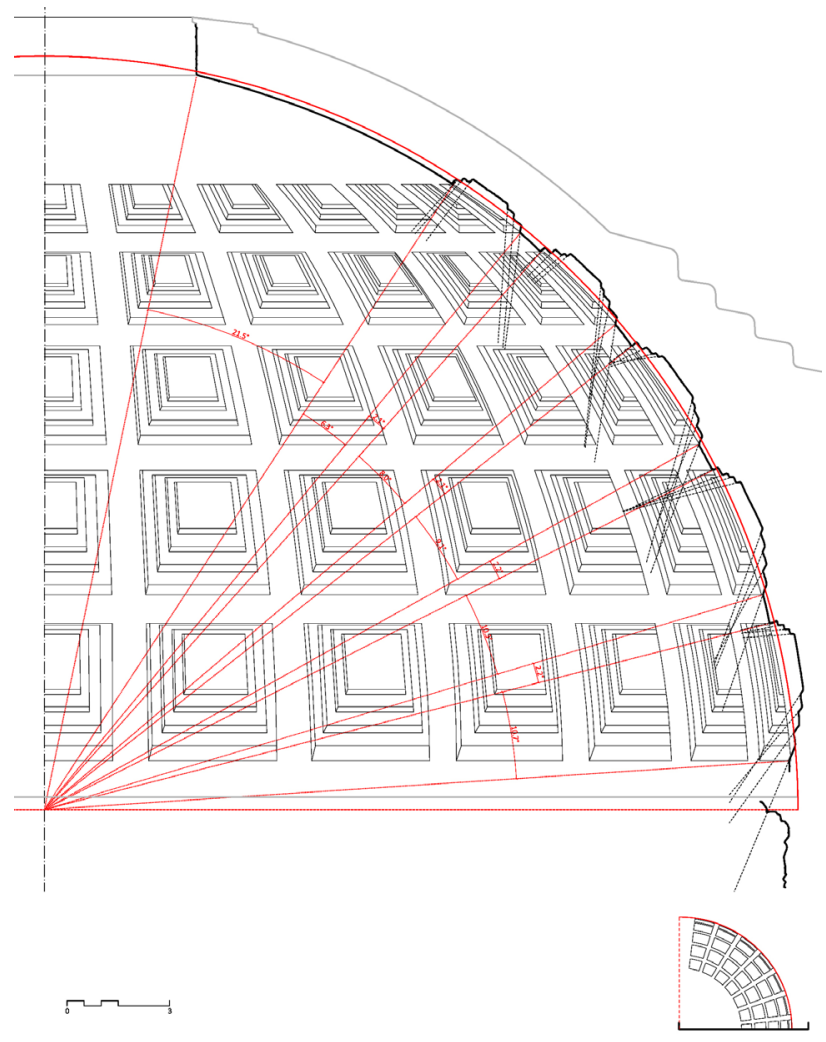

Figure 4. Study of a section of the Nord-East quadrant of the dome showing the offset between the cornice plan and the centre of the inscribed sphere. 


\subsection{Comparisons}

The point cloud analysis aims to detect and measure the differences between the survey results and the ideal model. Several graphical studies were realized to develop specific topics. The horizontal and meridian sections were overlapped to the curves of the ideal sphere. Some studies were applied directly on the 3D model. Many numerical controls join to the graphical analysis in order to produce quantifiable data.

These architectural analysis tools applied to the point cloud provide important and new data about the geometrical characteristics of the dome.

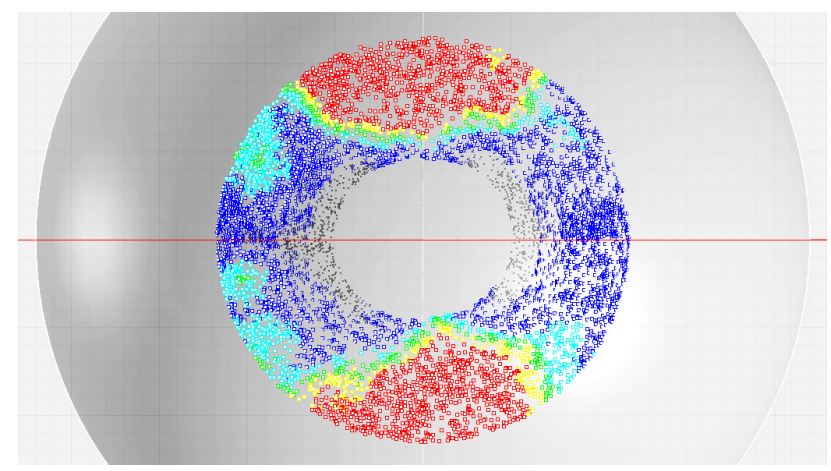

Figure 5. Gradation of distances from the points of the surveymodel to the ideal model on top of the dome.

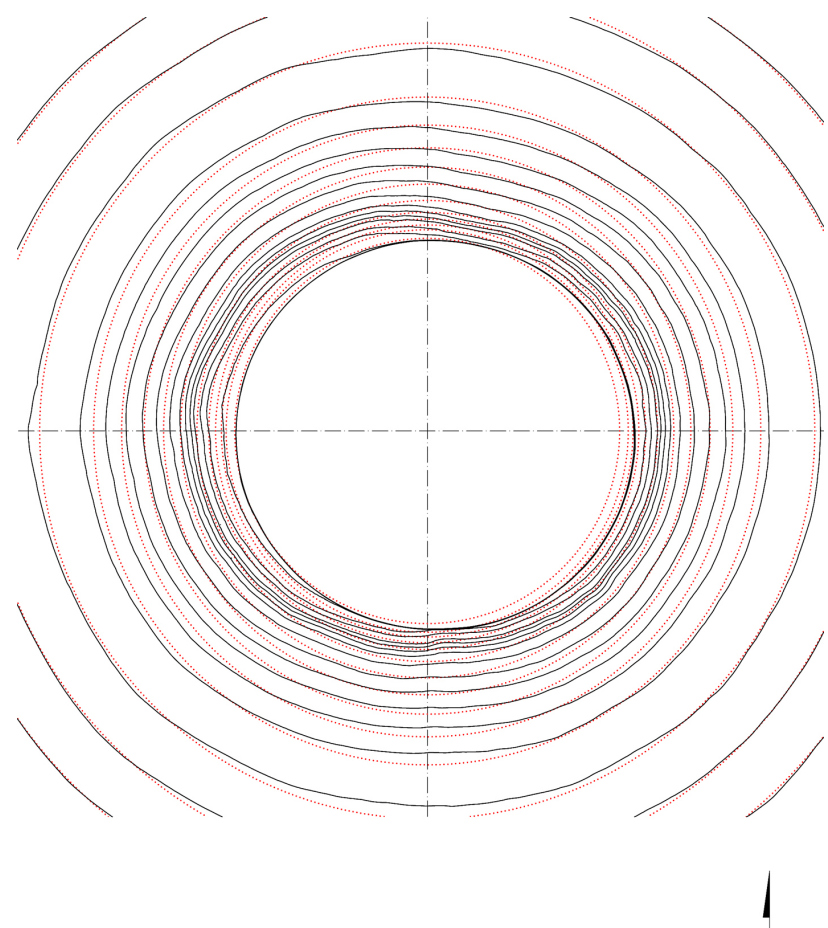

Figure 6. Horizontal sections of the upper area of the intrados of the dome: comparison between the survey model (black) and the ideal model (red).
These studies show the almost complete overlapping of the inner surface with the geometrical ideal model, except in a limited deformation area. The good conditions of the spheric shape are surprising, mostly considering its size and age.

In order to calculate the distance of each point to the ideal surface, a reference system with the origin in the centre of the average sphere was established. The data of the outcome show a $10 \mathrm{~cm}$ tolerance in $72.5 \%$ of the points, while $21 \%$ is under 15 $\mathrm{cm}$ and only $6.5 \%$ is located over this measure.

The results observation indicates that the greater number of points is very close to the spherical surface. The mean distance to the ideal sphere, calculated between all the points, is $7,8 \mathrm{~cm}$, that is the approximated equivalent of a $1 / 280$ of the average radius.

Regarding the separated parts of the dome the work states an average error of $5.5 \mathrm{~cm}$ in the coffers zone, which means a proportion of $1 / 400$ of the interior radius, while in the upper part, between the end of the coffers and the oculus it is $9.4 \mathrm{~cm}$. Data indicate in this part the existence of a special depression where the maximum deformation is held. In order to determine the exact location and dimension of this depression a specific analysis on this part has been properly detailed. As a result of the analysis of the cuts and level curves, data revel a deformation zone along the north-south axis of the dome emphasized in the upper level described before (Figg. 5 and 6).

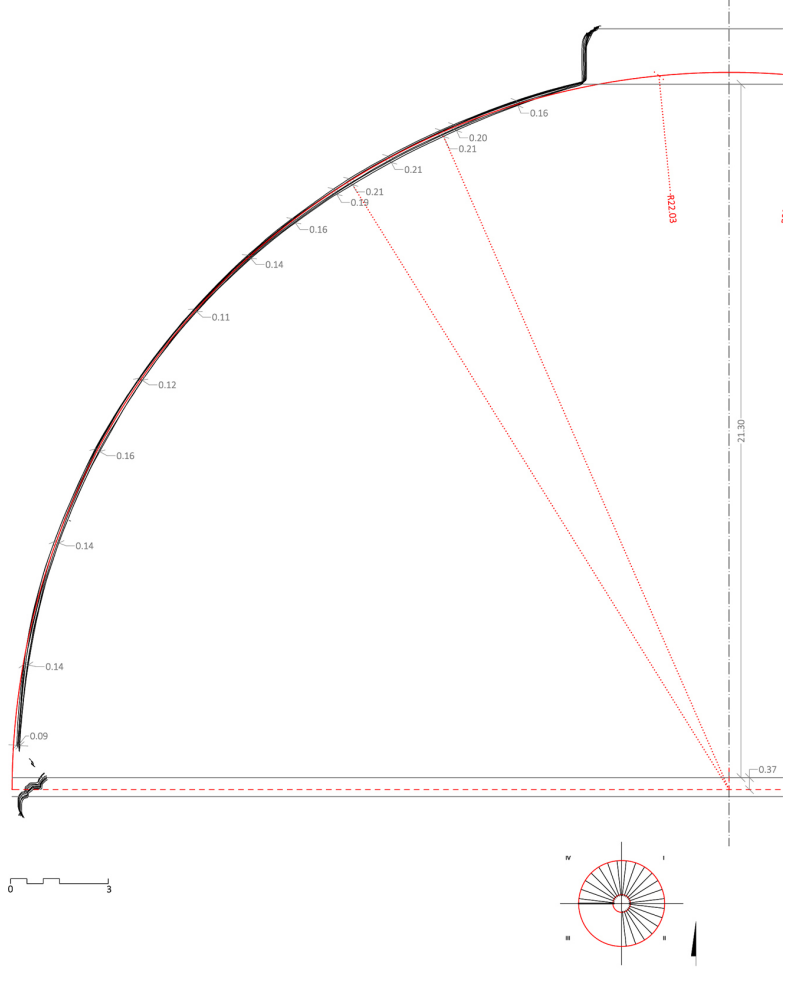

Figure 7. Study of the profile view of the dome by rotating the meridian sections of a quadrant of the dome in the same plan. 
Some recent studies indicate the existence of a deformation on the intrados of the dome, but they don't publish detailed information about this argument (Scherer 2009, Valenti 2009). With the purpose of calculating the dimension of that deformation we have analyzed in major quantity the distances of the survey points to the ideal sphere in this area. Part of the point cloud was selected applying a reduction of its density. This method allows determining the position and the extension of the deformed zone.

In order to realise the offset between the different profiles of the dome a rotation of the vertical sections has been applied. The vertical axis for this rotation has been positioned through the centre of the average sphere (Fig. 7).

The dome has been divided in four sectors according to the orthogonal axis of the plant view. Then a rotation has been applied to the curves of sectors I and III, to overlap with the lines of the sectors II and IV. The subsequent contrast of the projection of these lines with the average sphere shows de actual differences.

The maximum value of deviation in quadrant I equals $27 \mathrm{~cm}$, and it is found at the base of the dome along a 7 degree arch from the horizontal line. In Quadrant II this value stands at 17 $\mathrm{cm}$, and it is registered in the central-superior part of the dome.

The study of a sector of the scanner model can be of interest in order to provide a gross calculation without any of the common simplifications in data management. In this sense three meridian sections of the north-south sector have been produced. Two of these sections pass through the axis of the ribs and the third cuts the north-south axis through the coffer over the entrance to the building (Fig. 8). The sections of the point cloud in this part register a maximum distance to the ideal sphere of $0.32 \mathrm{~m}$ in the south and $0.20 \mathrm{~m}$ in the northern part next to the top.

The actual distribution of the coffers can also be the topic of an specific study. There is an evident complexity of design in their position. The partition of the sphere in 28 parts seams geometrically uncomfortable. The division of a quadrant in 7 parts was already a complex geometrical problem at that time.
The tool for the study of this distribution has been again the overlapping of the restitution model and the ideal one. In order to clarify the existence of actual alignments, orders and irregularities, we have used the orthogonal projection of the coffers' vertices. The use of informatics tools helped in the calculations of the average straight lines or arches, considering the results of both 3D scan and photogrammetry methods.

The analysis of the coffers provides additional information about their location. The carried out calculations demonstrate their extraordinarily precise distribution.

Their projection on the ground plan show the deviation of the bisecting lines of each individual sector compared to the axis of the ribs. The error registration stands at $0.6^{\circ}$. The maximum inaccuracy value in the most unfavorable axes equals $1^{\circ}$ and it is found in two of the 28 considered ribs. The angles between the axes of the sectors are extremely close to a precise value of $12.85^{\circ}$ that means it is close to the result of the subdivision of $360^{\circ}$ by 28 units.

The coffers are organized in 28 vertical sectors that contain five rows with a decreasing width. The construction lines passing through the external points of the coffers seem to be meridian circles of the maximum sphere inscribed. This study reveals how the vertical side meridians surprisingly do not converge in the centre of the oculus (Fig. 9). This indicates a reduced size of these circumferences, and leads to the idea that the construction actual axis were those cutting the coffers vertically through their midpoints in stead of those along the ribs' edges.

The two extensions of the outer lines of each coffer sector intersect in a vertex not coincident with the sphere pole. The distribution of these points does not describe a circle due to evident irregularities. Some of these vertexes approximate the pole while others separate from it significantly. The contrast between these outcomes with the geometrical analysis of the cupola shows specific deformations in the north and south parts of the dome. Indeed, only with a few exceptions, the existing vertexes in a longest distance to the pole are situated in those places.

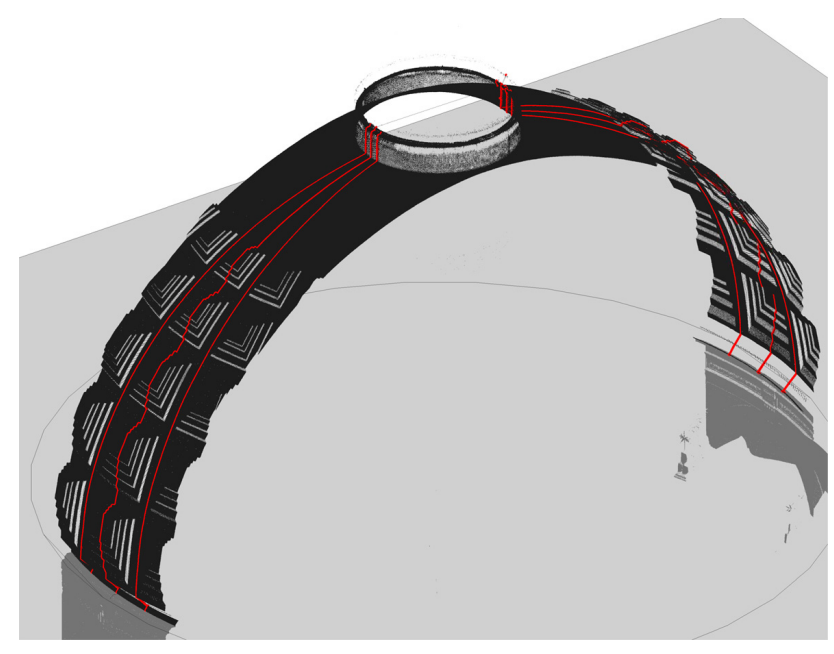

Figure 8. Meridian sections taken directly from the point cloud

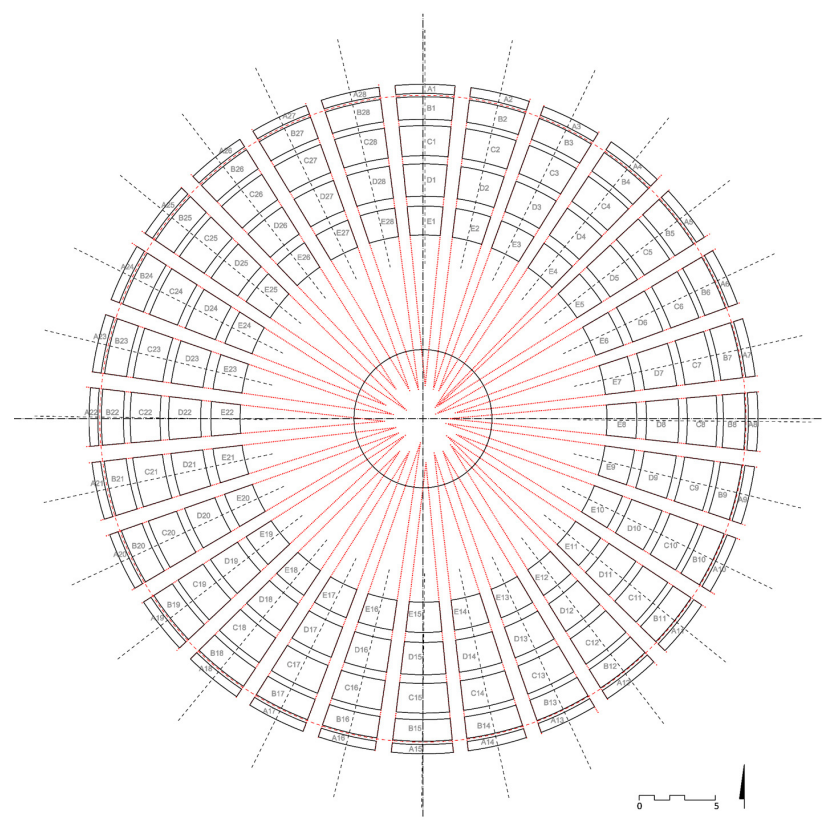

Figure 9. Alignments of the coffers' external edges showing that they don't converge in the center of the oculus. 


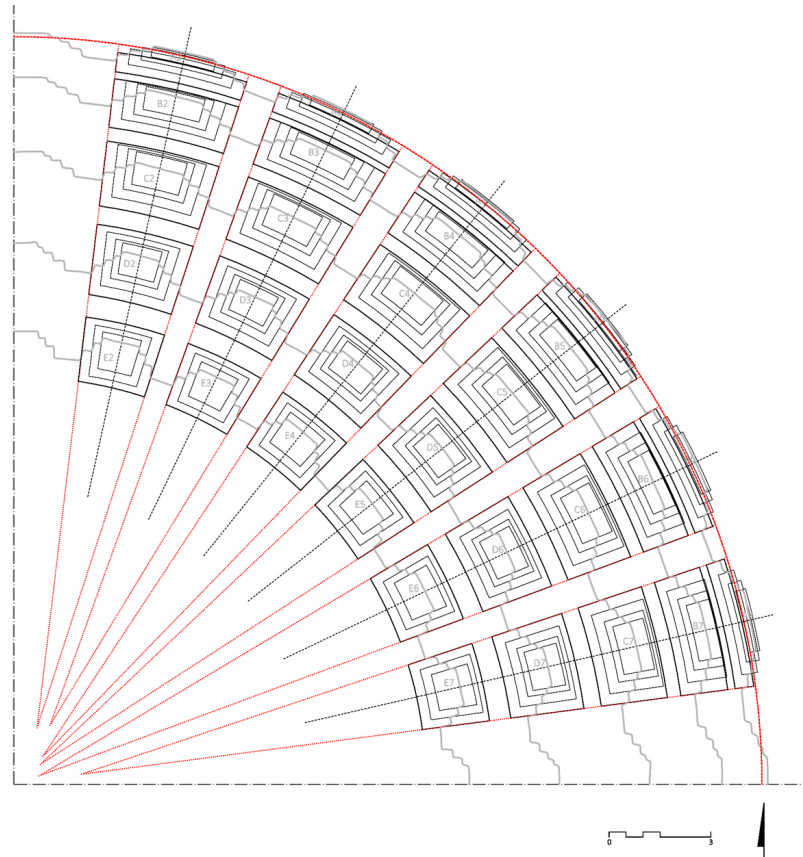

Figure 10. Study of the northeast quadrant of the dome with the convergent lines passing through the external vertices of the coffers.

\section{CONCLUSIONS}

This work shows the efficacy of an integrated survey that includes different methods of metric restitution and a comparative study of the documentation concerning the architectural object.

Some new information about the interior surface of the dome and some about the actual distribution of the coffers have been developed. The restitution of the present configuration of the intrados of the Pantheon can be used to study the current state of the dome and to determine its performance in time.

The system for the geometrical analysis and the data extraction from the point cloud that was applied can be considered as a relevant aspect of this research. The data selection and their treatment belong to a method based on the simplification of the information aimed to carry out specific analysis.

The comparison of the survey model and the ideal model was essential to develop the study. By using average calculations it was possible to determine their shape and relative position. The extraction of surfaces and curves from the point cloud and from the photogrammetric model was a necessary step in the research. This work shows like the bi-dimensional drawing is useful and effective to study the specific topics and to calculate detailed conditions of the object. The parallel analysis realized using three-dimensional models confirms and extends the results. The introduction of the comparison between the survey model and the ideal model can be a useful method for further architectural researches.

\section{ACKNOWLEDGEMENTS}

We would like to thank specially the willingness of Arch. Giovanni Belardi (curator of the Pantheon monument in the Soprintendenza per i Beni Architettonici e Paesaggistici per il

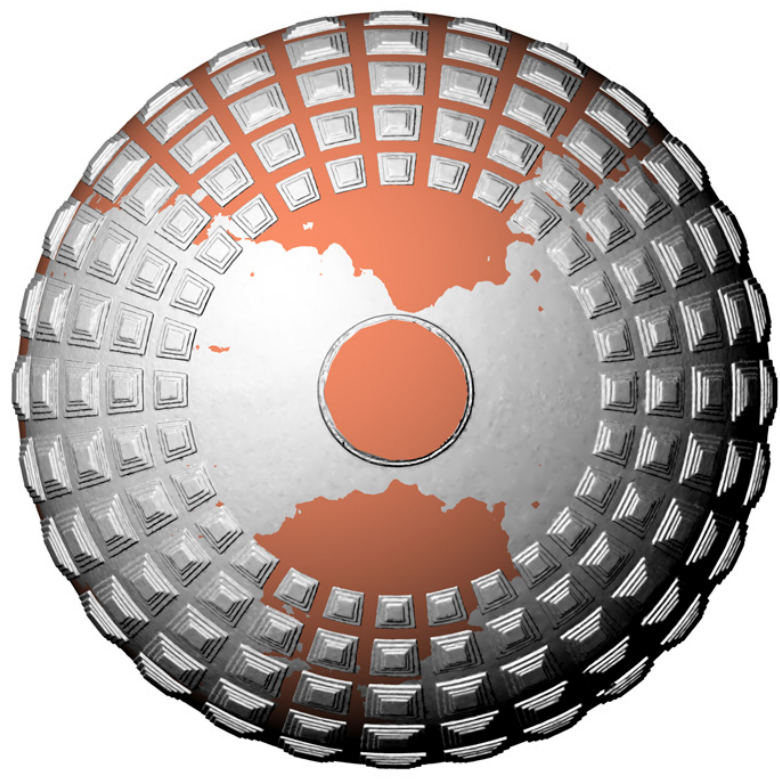

Figure 8. Comparison between the ideal model and the mesh surface extracted from the point cloud. Top view.

Comune di Roma). Also thanks to the Leica Geosystems delegation in Rome and specially to Arch. Valentina Albano for offering the instrumentation used to realize the laser scan survey.

\section{REFERENCES}

Aliberti, L., 2014. Pantheon y cupulas clásicas romanas: geometría y construcción. PhD. Universidad Politécnica de Madrid.

Aliberti, L., Altozano García, F., 2011. Documentación gráfica mediante fotogrametría digital de la cúpula del Pantheon en Roma. Actas del VII Congreso Nacional de Historia de la Construcción, Santiago de Compostela, pp. 33-43.

Belardi, G. 2006. Il Pantheon. Storia, tecnica e restauro. BetaGamma, Viterbo.

Canciani, M., Falcolini, C., Saccone, M., \& Spadafora, G. 2013. From point clouds to architectural models: algorithms for shape reconstruction. In: The International Archives of the Photogrammetry, Remote Sensing and Spatial Information Sciences, Trento, Italy, Vol. XL-5/W1, pp. 27-34.

Canciani, M., Sacccone M. 2011. The use of 3D models in integrated survey: the church of St. Thomas of Villanova in Castel Gandolfo. The International Archives of the Photogrammetry, Remote Sensing and Spatial Information Sciences, Trento, Italy, Vol. XXXVIII-5/W16, pp. 591-7.

Desgodetz, A. 1682. Les edifices antiques de Rome. 1779th ed. Claude Antoine Jombert fils, Paris. 
Docci, M., Maestri, D. 2010. Manuale di rilevamento architettonico e urbano. Laterza, Bari.

Fine Licht, K.d., 1968. The Rotunda in Rome. A Study on Hadrian's Pantheon. Nordisk Forlag, Copenhagen.

Geertman, H. 1980. Aedificium caeleberrimum. Studio sulla geometria del Pantheon. BA Besch, 55, pp. 203-26.

Graßhoff, G. 2009. The Bern Digital Pantheon Project. Plates. Bern Studies, Bern.

Lucchini, F. 1996. Pantheon. Carocci, Roma.

Pelletti, M. 1989. Note al rilievo del Pantheon. Quaderni dell'Istituto di Storia dell'Architettura, vol. XIII, pp. 10-18.

Scherer, M. 2009. A sustainable geodetic network for documenting and monitoring the Pantheon. In: The Pantheon in Rome. Contributions to the Conference Bern, November 9-12, 2006, pp. 215-22.

Valenti, G. M. 2009. A Computing Model for the Pantheon's Cupola: From the Discrete to the Continuous. The Ideal Continuous Model. In: The Pantheon in Rome. Contributions to the Conference Bern, November 9-12, 2006, pp. 223-233.

Waddell, G. 2008. Creating the Pantheon : Design, materials, and construction. "L'ERMA" di Bretschneider, Roma.

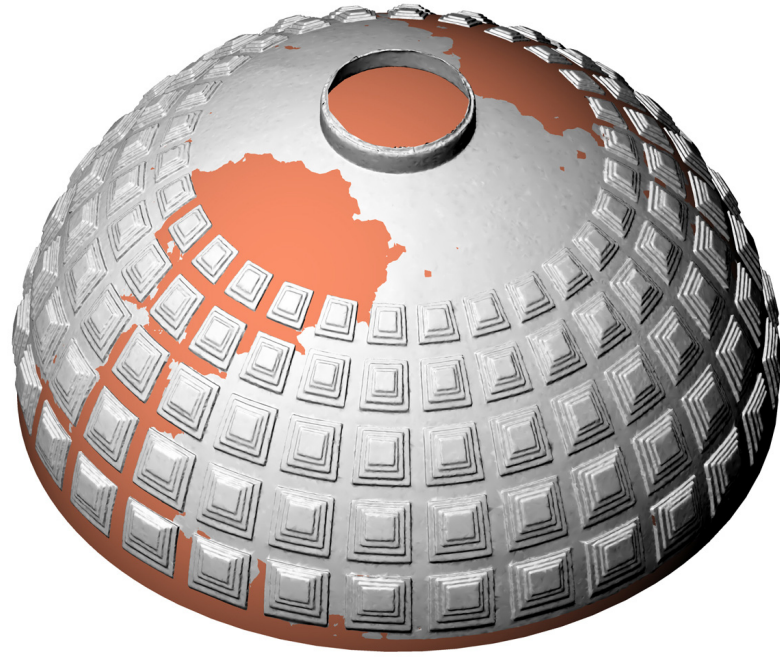

Figure 8. Comparison between the ideal model and the mesh surface extracted from the point cloud. Axonometric view. 